\title{
Application of inter-SINE amplified polymorphism (ISAP) markers for genotyping of Cucumis melo accessions and its transferability in Coleus spp.
}

\author{
SITI YULI MEILANDA SORMIN ${ }^{1}$, AZIZ PURWANTORO ${ }^{2}$, AGUS BUDI SETIAWAN", ${ }^{2, \boldsymbol{r}}$, CHEE HOW TEO ${ }^{3}$ \\ ${ }^{1}$ Department of Agronomy, Faculty of Agriculture, Universitas Gadjah Mada. Jl. Flora, Bulaksumur, Sleman 55281, Yogyakarta, Indonesia \\ ${ }^{2}$ Laboratory of Genetics and Plant Breeding, Department of Agronomy, Faculty of Agriculture, Universitas Gadjah Mada. J1. Flora, Bulaksumur, Sleman \\ 55281, Yogyakarta, Indonesia. Tel./fax.+62-274-551228, `email: setiawanab@ugm.ac.id \\ ${ }^{3}$ Centre for Research in Biotechnology for Agriculture, Universiti Malaya. Kuala Lumpur 50603, Malaysia
}

Manuscript received: 25 January 2021. Revision accepted: 28 April 2021

\begin{abstract}
Sormin SYM, Purwantoro A, Setiawan AB, Teo CH. 2021. Application of inter-SINE amplified polymorphism (ISAP) markers for genotyping of Cucumis melo accessions and its transferability in Coleus spp. Biodiversitas 22: 2918-2929. An unambiguous characterization of melon (Cucumis melo L.) accessions based on their morphological traits is often laborious and affected by environment when compared with molecular marker genotyping. Short interspersed nuclear elements (SINEs) are highly abundant nonautonomous and non-coding retrotransposons that are widely scattered over all chromosomes of eukaryotes. They can serve as a good molecular marker for routine genotyping in plant breeding and marker-assisted selection. This study aimed to apply inter-SINE amplified polymorphism (ISAP) markers for genotyping of Cucumis melo accessions and its transferability in Coleus spp. Twenty-one C. melo accessions, one C. metuliferus E. Mey. ex Naudin, and 15 accessions of Coleus spp. were used for ISAP marker development. A copy of cucumber-specific long interspersed nuclear element (LINE) and multiple copies of melon-specific SINE were identified and isolated. ISAP primers were designed from the highly conserved region of the SINEs and LINE. The melon and cucumber-specific ISAP markers showed a higher degree of polymorphism (87.5\%-100\%) than potato ISAP markers $(60 \%-100 \%)$ in all the tested melon accessions. The unweighted pair group method with arithmetic average (UPGMA) dendrogram generated from polymorphic ISAP bands clearly distinguish the Cucumis melo accessions from their distantly related wild species $C$. metuliferus. The transferable nature of Cucumis ISAP marker system was demonstrated in Coleus species, where the marker differentiates the tested accessions.
\end{abstract}

Keywords: Coleus, Cucumis melo, ISAP marker, plant genotyping, SINE elements

\section{INTRODUCTION}

Melon (Cucumis melo L.) is an economically important horticultural crop in the genus Cucumis. It is a diploid species with $2 \mathrm{n}=2 \times=24$ chromosomes (Setiawan et al. 2020a). Over the years, various melon varieties with distinct traits, stable yields, and uniform phenotypes have been developed by different melon breeding programs. In addition, numerous pre-breeding accessions exist, including the landraces and their wild accessions maintained by various gene banks around the world (Sebastian et al. 2010; Pitrat 2016). These melon accessions show a wide range of genetic diversity in their fruit size and shape, skin color, flesh color, seed characteristic, nutritional value, and floral diversity (Pandey et al. 2021). Various morphology and agronomic characters have been widely used for the selection of inbred LINEs and phenotyping of melon cultivars (AliShtayeh et al. 2017; Merheb et al. 2020). However, the selection remains a laborious task because of the high number of melon cultivars (Pitrat 2016) and the phenotypic traits are often affected by environments. Molecular marker genotyping, which is less influenced by environmental factors, provides a reliable solution to phenotypic characterization.
The use of molecular markers in melon genotyping has been extensively studied. Random amplified polymorphism DNA (Karimi et al. 2016), sequence-related amplified polymorphism (Yildiz et al. 2011), chloroplast DNA marker (Rodríguez-Moreno et al. 2011), and internal transcribed spacer of ribosomal DNA (Renner et al. 2007; Endl et al. 2018) have been used to study the genetic diversity and domestication processes in melon. In addition, simple sequence repeat (SSR) markers have been applied in melon genetic diversity studies and genetic map development (Raghami et al. 2014; Zhu et al. 2016). However, RAPD is poorly reproducible, and the other markers are costly and require many combinations of SSR markers to obtain reliable results.

Retrotransposons are classified into two subclasses: long terminal repeat (LTR) retrotransposons, including Ty1/Copia and Ty3/Gypsy families, and non-LTR retrotransposons, including long interspersed nuclear element (LINE) and short interspersed nuclear element (SINE) families (Schulman et al. 2012; Elbarbary et al. 2016; Orozco-Arias et al. 2019). SINE elements are highly abundant non-LTR retrotransposons that are about $100 \mathrm{bp}$ to $500 \mathrm{bp}$ in length and show high sequence diversity (Wenke et al. 2011; Seibt et al. 2016). SINE has retrotransposition ability via an RNA intermediate, where new copies of the elements will transpose and integrate at 
other genomic locations while maintaining their original location. Therefore, SINE is suitable for molecular marker development. Wenke et al. (2015) successfully developed inter-SINE amplified polymorphism (ISAP) marker for plant genotyping. The ISAP markers developed by various groups produced genotype-specific fingerprints at high resolutions, thus allowing cost-efficient, robust, and rapid plant genotyping (Seibt et al. 2012; Wenke et al. 2015; Diekmann et al. 2017; Pantchev et al. 2019).

The application of ISAP markers in melon genetic diversity and genotyping study is still limited. In addition, information on the transferability of the ISAP marker in other plant species is scarce. This study aimed to apply inter-SINE amplified polymorphism (ISAP) markers for genotyping of Cucumis melo accessions and its transferability in Coleus spp.

\section{MATERIALS AND METHODS}

\section{Plant materials}

Twenty-one Cucumis melo accessions, one $C$. metuliferus E. Mey. ex Naudin (Table 1), and 15 accessions of Coleus spp. were used in this study. The accessions of Coleus species (Col-K01, Col-K02, Col-K03, Col-L01 ColL02, Col-L03, Col-M01 Col-M02, Col-M03, Col-N01 ColN02, Col-N03, Col-O01 Col-O02, and Col-O03) were collected from the Yogyakarta region and maintained in the Genetic and Plant Breeding Laboratory, Faculty of Agriculture, Universitas Gadjah Mada. The seeds of $C$. melo accessions were germinated in moistened pot trays, grown, and maintained in the screen house of the
Department of Agronomy, Faculty of Agriculture, Universitas Gadjah Mada, Indonesia, from April until July 2019.

\section{DNA isolation and quantification}

Total genomic DNA was extracted from the young leaves of Cucumis accessions and Coleus spp. By using the CTAB (hexadecyltrimethylammonium bromide) extraction method described by Setiawan et al. (2020a), DNA quantification was conducted with NanoDrop (2000c Spectrometer, Thermo Scientific). The DNA samples of each accession were diluted to a working solution (25 $\mathrm{ng} / \mu \mathrm{l})$ with nuclease-free water.

\section{LINE and SINE sequences analysis}

Cucumber LINE (CsLINE-1) was retrieved from the genome database of Cucumis sativus cv. 9930 by searching the corresponding database with the keywords "LINE" and/or "long interspersed nuclear element." SINE sequences were retrieved from melon and cucumber genomes by using the local blastN approach with CsLINE1 as the search query. Sequence synteny analysis was performed by comparing the sequences of SINE elements (CsSINE-1, CmSINE-1, and CmSINE-2) in melon and cucumber genomes with CsLINE-1 in cucumber. Phylogenetic analysis of CsSINE-1 was conducted using MEGA 7 (Kumar et al. 2016) with the neighbor-joining (NJ) method with 1,000 bootstrap replicates. Insertion sites and copy numbers of CsSINEs inserted into cucumber genes were determined using blastN analysis against reference genome (C. sativus cv. 9930).

Table 1. Plant materials used in this study

\begin{tabular}{|c|c|c|c|}
\hline Name/code & Accession number/status & Species & Origin \\
\hline Golden Langkawi & Commercial variety & Cucumis melo L. & Malaysia \\
\hline Silver Light & Commercial variety & Cucumis melo $\mathrm{L}$. & Japan \\
\hline Melani & Commercial variety & Cucumis melo $\mathrm{L}$. & Indonesia \\
\hline Japonica & Commercial variety & Cucumis melo $\mathrm{L}$. & Indonesia \\
\hline Rock Sonya & Commercial variety & Cucumis melo $\mathrm{L}$. & Indonesia \\
\hline Dainty & Commercial variety & Cucumis melo $\mathrm{L}$. & Indonesia \\
\hline Eksis & Commercial variety & Cucumis melo $\mathrm{L}$. & Indonesia \\
\hline Baladewa & Commercial variety & Cucumis melo $\mathrm{L}$. & Indonesia \\
\hline US340 & PI 185111 & Cucumis melo $\mathrm{L}$. & Ghana \\
\hline US143 & PI 292190 & Cucumis metuliferus E.Mey. ex Naudin & South Africa \\
\hline Madesta F1 & Commercial variety & Cucumis melo $\mathrm{L}$. & Indonesia \\
\hline Haru & PI 266944 & Cucumis melo $\mathrm{L}$. & England \\
\hline NI19 & 940281 & Cucumis melo L. & Chad \\
\hline P34 & Weedy melon & Cucumis melo L. & Korea \\
\hline P107 & Rio Gold & Cucumis melo L. & USA \\
\hline US176 & PI 614576 & Cucumis melo L. & India (Center) \\
\hline US128 & PI 482398 & Cucumis melo L. & Zimbabwe \\
\hline US205 & PI 182952 & Cucumis melo L. & India (West) \\
\hline US58 & PI 116738 & Cucumis melo L. & India (West) \\
\hline US368 & PI 505599-1 & Cucumis melo L. & Zambia \\
\hline US54 & PI 614588 & Cucumis melo L. & India (Center) \\
\hline US171 & PI 614542 & Cucumis melo L. & India (Center) \\
\hline
\end{tabular}


Table 2. ISAP primers used in this study

\begin{tabular}{llccc}
\hline \multicolumn{1}{c}{ Primer name } & \multicolumn{1}{c}{ Sequence $\left(\mathbf{5}^{\prime} \mathbf{- \mathbf { 3 } ^ { \prime } )}\right.$} & Tm $\left({ }^{\circ} \mathbf{C}\right)$ & GC $(\boldsymbol{\%})$ & Reference \\
\hline CmLINE_ISAP1 & GAADRGTGTGAGGAGGAAGYTVTG & 66.2 & 50.0 & This study \\
CmLINE_ISAP2 & CCAYTTKTCARMRTCACCCACC & 68.7 & 52.3 & This study \\
CsSINE1-01* & GTCCNGTGAGATTAGTHGAGGTG & 63.9 & 51.4 & This study \\
CsSINE1-02 & GACAYBCAAATGTTGTAGGGTCAG & 66.1 & 46.5 & This study \\
CsSINE1-03 & TTACCACTAGGNCAACCCANGATGG & 70.9 & 52.0 & This study \\
SolS-IIIaF & CCTATGTGGTTTGCGAGC & 62.4 & 55.6 & Seibt et al. (2012) \\
SolS-IVR & CCCTTTGGATCAATCACAGC & 64.8 & 50.0 & Seibt et al. (2012) \\
\hline
\end{tabular}

Note: *) The ISAP primer was tested in Coleus spp.

Table 3. LINE and SINE elements found in cucumber and melon genomes

\begin{tabular}{llllcr}
\hline \multicolumn{1}{c}{ Name } & \multicolumn{1}{c}{ Accession } & \multicolumn{1}{c}{ Position } & \multicolumn{1}{c}{ Start-End } & Length (bp) & Target site duplication \\
\hline CsLINE-1 & NC 026661.1 & Chromosome 7 & $15506082-1528378$ & 3153 & Yes \\
CsSINE-1 & NC 026658.1 & Chromosome 4 & $1535820-1536085$ & 260 & Yes \\
CmSINE-1 & NW 007546275.1 & scaffold00008 & $72133-72388$ & 241 & Yes \\
CmSINE-2 & NW 007546313.1 & scaffold00046 & $1527032-1528378$ & 1347 & Yes \\
\hline
\end{tabular}

\section{ISAP primer design and PCR assay}

Multiple sequence alignment (MSA) of cucumber and melon SINE and LINE sequences were performed by using Bioedit (Hall 1999). Cucumber- and melon-specific ISAP primers were designed using FastPCR from a consensus sequence generated from highly conserved regions of cucumber-specific SINE and melon-specific LINE sequences (Table 2) (Kalendar et al. 2017). For PCR amplification, $50 \mathrm{ng}$ of genomic DNA was added to a reaction mixture that consists of $1 \mathrm{X}$ GoTaq ${ }^{\circledR}$ Green Master Mix (Promega, USA), $0.2 \mathrm{mM}$ dNTPs, $0.2 \mu \mathrm{M}$ primer, $1.25 \mathrm{U} / \mu \mathrm{L} \mathrm{GoTaq}{ }^{\circledR}$ polymerase, and nuclease-free water in a $12.5 \mu \mathrm{L}$ reaction volume. The amplification conditions are initial denaturation at $95{ }^{\circ} \mathrm{C}$ for $5 \mathrm{~min}$; 30 cycles of $95^{\circ} \mathrm{C}$ for $1 \mathrm{~min}$; annealing at the $\mathrm{T}_{\mathrm{a}}$ specified in Table 3 for $1 \mathrm{~min}, 72^{\circ} \mathrm{C}$ for $1 \mathrm{~min}$; and a final extension at $72^{\circ} \mathrm{C}$ for 7 min. The amplification was performed using T100 $\mathrm{TM}$ thermal cycler (Bio-Rad, USA). PCR products were analyzed on $2.5 \%(\mathrm{w} / \mathrm{v})$ MetaPhor $^{\mathrm{TM}}$ (Lonza, USA) agarose gel.

\section{Data analysis}

The presence and absence of ISAP bands were scored as 1 and 0 , respectively. Clustering analysis of ISAP bands was performed using NTSYS-PC software (Rohlf 2009). The SIMQUAL program was used to calculate Jaccard's similarity coefficient and a dendrogram was generated by the mean of the unweighted pair group method with arithmetic average (UPGMA) method.

\section{RESULTS AND DISCUSSION}

\section{Analysis of truncated SINE elements originated from Cucumber LINE sequences}

One copy of LINE (CsLINE-1) was identified from the cucumber genome (C. sativus cv. 9930). CsLINE-1 is a non-autonomous LINE as it contains only the reverse transcriptase (RVT_2) domain and lacks the GAG and AP domains. With the use of CsLINE-1 sequence as a homology search query for blastN analysis, multiple copies of SINEs (CmSINE-1, CmSINE-2, and CsSINE-1) were retrieved from the genomes of melon and cucumber. Sequence synteny analysis in this study allows clear identification of the SINE sequence features when comparing with CsLINE-1. SINE-1 sequences are highly conserved in both cucumber and melon genomes, while CsLINE-1 is found in the cucumber genome only. For CmSINE-2, most of the important retrotransposon domains such as GAG, AP, and RT domains were missing from the element (Figure 1). Detailed analysis of the melon and cucumber genomes found no evidence of a full-length autonomous copy of CsLINE-1 in these genomes. This finding indicates that CsLINE-1 was truncated and the autonomous copy was lost from the melon and cucumber genomes before the divergence of these two genomes.

Two different types of SINE derived from CsLINE-1 were detected in the melon genome (CmSINE-1 and CmSINE-2), whereas only one type (CsSINE-1) was found in the cucumber genome (Figure 1). CsLINE-1, CsSINE-1, CmSINE-1, and CmSINE-2 are 3153, 260, 241, and 1347 bp in length, respectively. All elements contain target site duplication at the $5^{\prime}$ and $3^{\prime}$-end of their sequences. The details of these sequences, including the accession numbers where these elements are located and their positions, are listed in Table 3. An NJ dendrogram was constructed from 131 copies of CsSINE-1 sequences retrieved from $C$. sativus cv. 9930. The NJ dendrogram divided CsSINE-1 sequences into 8 clades, i.e., clades A, A1, A2, B, C, D, D1, and E (Figure 2). The largest clade of CsSINE-1 consists of 30 members (D1), while clades A, A1, A2, B, $\mathrm{C}, \mathrm{D}$ and $\mathrm{E}$ consist of 10,10, 24, 14, 13, 13 and 11 members, respectively. 


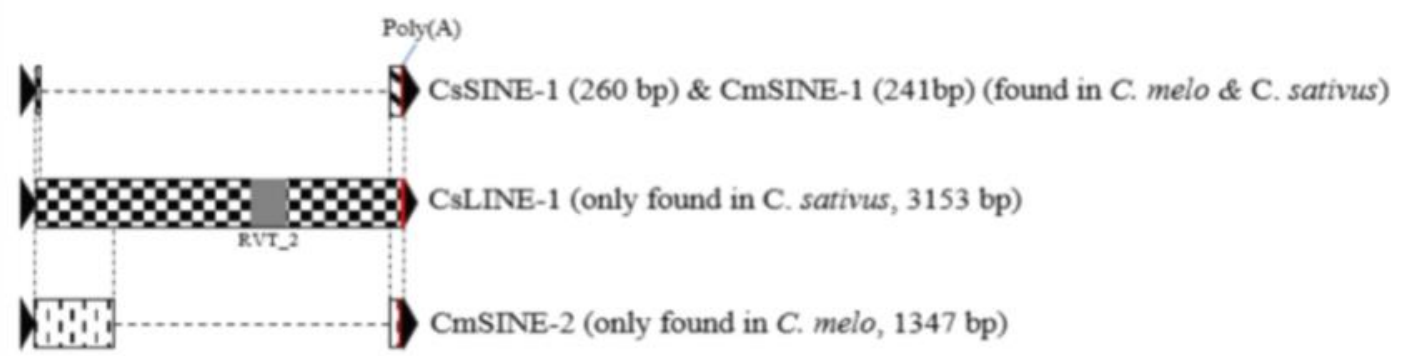

Figure 1. Sequence synteny analysis of cucumber-specific-LINE element (CsLINE-1) and its corresponding SINE elements (CsSINE-1, CmSINE-1, and CmSINE2) retrieved from cucumber and melon genomes. The CsLINE-1 contains target site duplication (arrowheads) at upstream and downstream, $3^{\prime}$ poly A-tails, and the reverse transcriptase domain but lacks other retrotransposon domains.

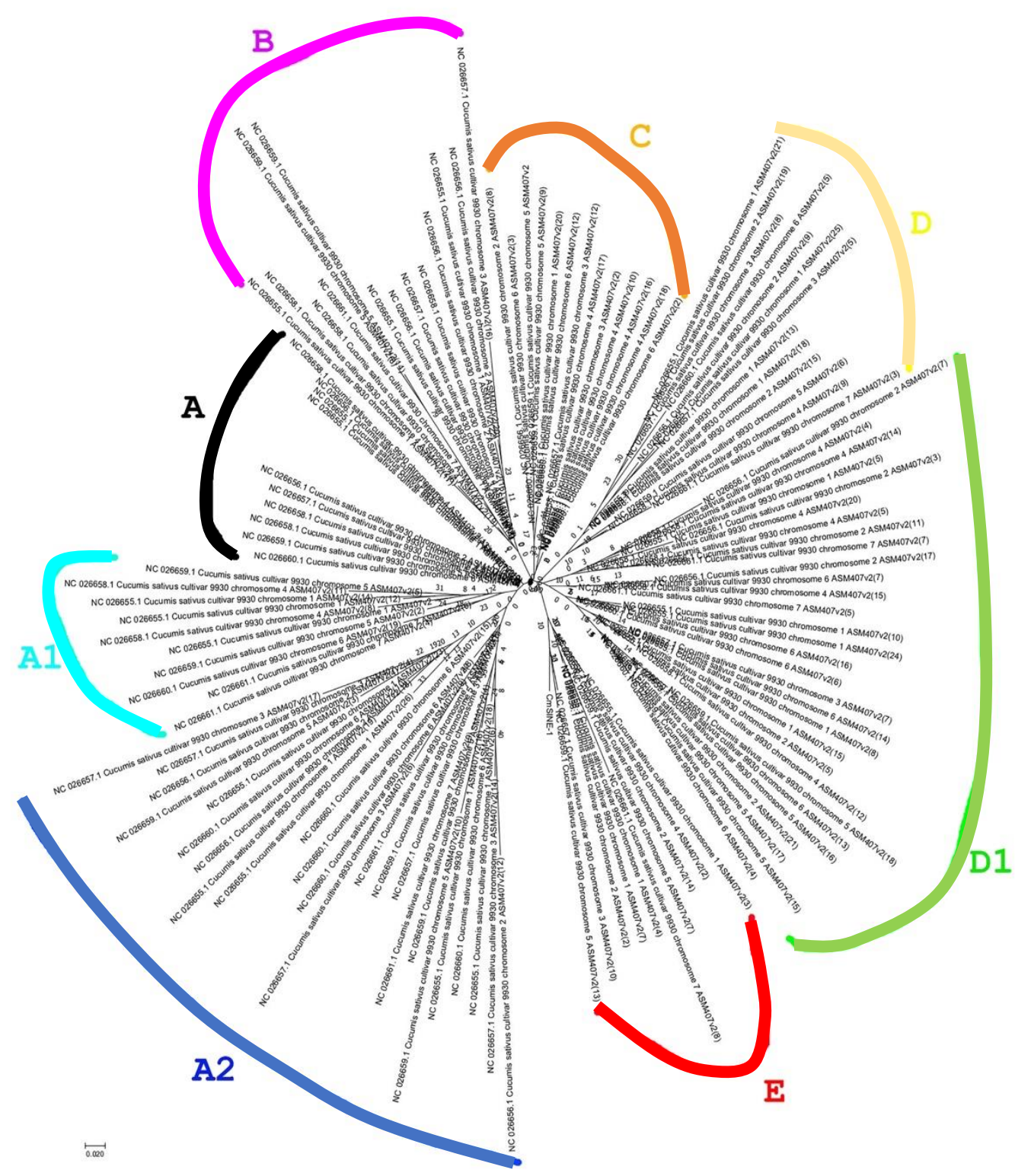

Figure 2. NJ tree of CsSINE-1 elements. The optimal tree with the sum of branch length $=5.38336563$ is shown. The tree is drawn to scale, with branch lengths in the same units as those of the evolutionary distances used to infer the phylogenetic tree. The evolutionary distances were computed using the Jukes-Cantor method and are in the units of the number of base substitutions per site. The analysis involved 126 nucleotide sequences. All positions containing gaps and missing data were eliminated. The final dataset contained a total of 23 positions. Evolutionary analyses were conducted in MEGA7. 


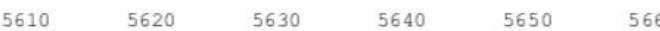

LN713257.1:22004281-22010938 C LN713261 . 1 : C9255509-9248886 Cu NW_007546309.1: c3570407-35673 LN6 $61893.1: c 1817877-1811241 \mathrm{Cu}$ NW_007546282.1:2699869-2702866 NW_007546290.1: c1256313-125323 NW_007546282 .1:835029-838562 CmLINE1-Un-D3 NW-007546271.1 CmLINE1-Un-D4 NW-007546347.1 CmLINE1-Un-D5 NW 007546306 , CmLINE1-Un-D6 NW-007546322, CmLINE1-Un-D7 NW 007546309. CmLINE1-Un-D8 NW 007546368 . CmLINE1-Un-D9 NW 007546290.1 CmLINE1-Un-D10 NW̄_007546354.1 CmLINE1-D11 NW $00 \overline{7} 546307.1$ CmLINE1-6-D1 LN $713260.1: 122315$ LN713257.1: c9964803-9958162 C NW_007546328 Consensus CmLINE1-ISAP1

CCTCTCATAGGATTGAGATTGAGGGTTGAGTGTCTTGGATTGTCATCAGGGTATTGG AAGGGTGTGAGAGGAAGTTATGGCGCGTTCTTGG CCTCTCACAAGATTGGGTATTCGGGAGTTGATTTGTCTAGAATTTGTCATTAGGGTATTGGG AAGGGTGTGAGGAGGAAGTTGTGACACGTTCTTTGG CCTCTCATAGGATTGGGCATTGGGGGTTGAGTTGTCTTGGATTTGTCATCGGGGTATTGG AAAGGTGT GAGAGGAAGTTGTGGCGGTTCTTTGG CCTCTCATAGGATTGGACATTGGGGGTTGAGTTGTTTTGGATTTGTCATCAGGGTATTGGG-AAGGGTGTGAGGAGGAAGCTGTGGCGCGTTCCTTGG CCTCTCATAAGATTGGGTACTGGGGGGTTGAGTTGTCT TAGATT TGTCACCAGGGTATTGGG AAGGGTGTGA GAAGAAGTTATGACGCGTTTTTTGG CCTCTTACAGGATTAGGTATTGGGGGGTTAGTTGTCTTGAATTTGTCGTCAAGGTATTGGA-AATGGTGT GAGGAGGAAGTTGTGGTGGGTCATTGG CCTCACATAGGATTGGGATTGGGGGTGGAGTTATCTTGGATTTGTCATCAGGGTATTAGG-AAGGGTGTGAGAGGAAGCTGTGGCGCGTCTTTGG? CCTTACATAGGATCAGAGGATGAGATGTTGAGTTGTCTTAGATTT-CTGTAAAGGTATTAGG-AAGGATGTGA GGAGGAAGCTGTGGTGTGTGCTCTAG СCTCTCATAAGATTGGGAGTGGGATGTTGAGTTGTCTTGGGTTTGCCATCAA-DTAGG AAGGGTGTGAGAGGAAGCT GTGGGGTGTGCTCTGG? TCTCTCACAGGATTAGGTAT TGAGGGGT TGAGTTGTCTTGGATTTGTCGTCAGGGTATTAGG AAGAGTGTGAGAGGAAGCTGTGCCACATTCTCTGG CCTCTCATAGGATTGGGATTGGAGGGTTGAGTTGTCTTGGATTTGTCATTAAGGTATTGGG AAGGGTGTGAGGAGGAAGCTGTGACGTGTTCTTTGG CCTCTCATAGGATTGGGCATTGGGGGGTTGAGTTCTCTTGGATTTGTCATCAGGGTATTGGG AAAGGTGTGAGGAGGAAGTTGTGGCGCTTTTGTGG CCTCTTATAGGATTGGGATTGGGGGGTTGAGTTGTCTTGGATTTTTCATCAGGGTATTGG -AAGGGTGTGAGGAGGAGTTGTGGCGCTTCTTTGG ССTCTTATPGGATAGGCATT GAGGGGTGATTGTCTTGGATTTGTCATCAGGGTATTGA-AAGGGTGT GA GAAGAAGTTGTGGCGAGTCTTTGG CTTCTCATAGGATCAGGTATTGGGGGGT TGAGTTGTCT TGGATTTGTCATCAGGG TATTGGG - ATAAGT GT GAGGAGGAAGCTATGGCGCGTTCTITTG GTATTGAA-AAGGGTGTGAGAGGAATTGTGGCACGTTCTCTAG CCTCTCATAGGATTGAGCATTGAGGGGTTGAGTTGTCTTGGATTTGTCATCAGGGTATTGGG-AAGGGTGTGAGGAGGAGTTATGGCGCGTTCTTTGGT CTTTTCACAAAATTGGGTATTGGGGGGT TAGTTGTCTTTGATTTGTCGTTAGGGTATTGGGAAGGGT GT GAGGAGGAAGTT GT GGCGTGTTCTCTGG CCTCTCATAGGATTGTGCATTGGGGGGTTAGTTGTCTTGGATTTGTCATCAAGGTATTGGG-A-GAGTGTGAGGAGGAAGCTCTGGCGTGTTCTTTGG CYTYWYAYARGA TYRD RNRNTGRRRD GTTGAGTTVTCTTDRATTTKYCRTHARGGTATTDGRGAADRGTGTGAGGAGAAGYTVTGVBRNGTKYHBTDG CYTYWYAYARGATYRD RNRNTGRRRDGTTGAGTTVTCTTDRATTTKYCRTHARGGTATTDGRGAADRGTGTGAGGAGGAAGYTVTG

Figure 3. MSA of LINE elements of C. melo. CmLINE-1 primers used for ISAP markers were designed from the conserved regions. Only one part of the MSA with one primer site (starts-ends: 5663-5685) is shown here due to the length of MSA, which reaches up to 6245 bp.

$$
110
$$

130

140

150

160

170

180

190

200

NC_026655.1 Cucumis sativus cu NC_026655.1 Cucumis sativus cu NC_026655.1 Cucumis sativus cu NC-026655.1 Cucumis sativus cu NC 026655.1 Cucumis sativus cu $\mathrm{NC}^{-026655.1}$ Cucumis sativus $\mathrm{Cu}$ NC 026655.1 Cucumis sativus cu NC-026656.1 Cucumis sativus cu NC_026656.1 Cucumis sativus cu NC_026656.1 Cucumis sativus cu NC-026656.1 Cucumis sativus cu NC-026656.1 Cucumis sativus NC_026656.1 Cucumis sativus cu
NC-026657.1 Cucumis sativus cu NC_026657.1 Cucumis sativus cu
NC-026657.1 Cucumis sativus cu NC-026657.1 Cucumis sativus cu NC-026657.1 Cucumis sativus cu NC_026657.1 Cucumis sativus cu NC-026657.1 Cucumis sativus cu NC-026657.1 Cucumis sativus cu NC_026657.1 Cucumis sativus cu NC_026657.1 Cucumis sativus cu NC-026657.1 Cucumis sativus cu NC_026657.1 Cucumis sativus cu NC_026657.1 Cucumis sativus cu NC_026657.1 Cucumis sativus cu NC-026658.1 Cucumis sativus cu NC-026658.1 Cucumis sativus cu NC 026658.1 Cucumis sativus NC_026658.1 Cucumis sativus cu NC_026658.1 Cucumis sativus cu NC_026658.1 Cucumis sativus cu NC_026658.1 Cucumis sativus cu NC-02658.1 Cucumis sativus Cu NC_026658.1 Cucumis sativus cu
NC-026658.1 Cucumis sativus cu NC_026658.1 Cucumis sativus Cu
NC-026658.1 Cucumis sativus cu NC_026658.1 Cucumis sativus cu
NC-026659.1 Cucumis sativus cu NC_026659.1 Cucumis sativus Cu NC_026659.1 Cucumis sativus cu
NC-026659.1 Cucumis sativus cu NC_026659.1 Cucumis sativus cu NC_026659.1 Cucumis sativus cu NC_ 2665.1 Cucumis sativus cu NC_026659.1 Cucumis sativus cu NC-026659.1 Cucumis sativus cu NC_026659.1 Cucumis sativus cu
NC-026659.1 Cucumis sativus cu NC_026659.1 Cucumis sativus cu
NC-026660.1 Cucumis sativus cu NC_026660.1 Cucumis sativus cu NC_026660.1 Cucumis sativus cu NC-026660.1 Cucumis sativus cu NC-026660.1 Cucumis sativus cu NC-026660.1 Cucumis sativus cu NC_026660.1 Cucumis sativus cu NC_026660.1 Cucumis sativus cu NC_026660.1 Cucumis sativus cu NC-026660.1 Cucumis sativus cu NC-026660.1 Cucumis sativus cu NC_026661.1 Cucumis sativus cu NC-026661.1 Cucumis sativus Cucus-I

Consensus

CsSINE1-01

CsSINE1-02

TAATTTCCTACGAGTTTCCTTCACATCCAAATATTGTAGGGTTAAGTGGGTTGTGCCGTGAGATTAGTTGAGGTGTGCGCAAGCTAGCCCAGACACTCAT TAATTTCCTACGGGTTTCCTTGACACCCAATTGTTGTAGGGTCAGGCGGGTTATCTCGTGAGATTAGTTGAGGTGCGCGTAAGCTGGCTCGGACACTCAC TAATTTCTTACGAGTTTCCT TGACACTCAAATGTTGTAGGGTCAGGCAGGTTGTCCCGTGAGATTAGTTGAGGTGCACGTAAACTAGCCCAAACACTCAC TAATTTTCTATGAGTTTCTTTTATACTCAAATGTTGTGGGGTCA GACGGGTTGTCCCGTGAAATT AGTTGA GA TGCTCGTAAGCTGGTCTGGACACTCAT TAATTTTCTATAAGGTTTCTTGACACCCAAATATTGTAGGGTCAG -_-_GTGGATTAGTCAAGGTGCGCGTAAGCTGGCCATACACTCAC

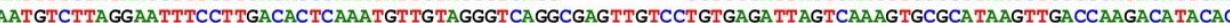
GAA- AATTTCCTTGATACCCAAATATTGATGGGTTAGAGGAGTTGTCCTGCTAGATTATTTGAGGTGTTCATAAGCTATTCTGAACACTCAC TAATTTCCTACGAGTTTTCTTGACACCCAAATATTGTAGGGTCA GACGGATT GACCCATAAGA TT AGTCGA TGTGCGCT TAAGTTGATCCAGACACTCAC ATTTCCTA TGAGTTTCCTTG-CACCCAAATGTTGTATGATCAGACGGGTTGTCCCGTGAGATTAGTCGAGGTGCGCATAAGCTGGCCCGAACACTCAC Ta

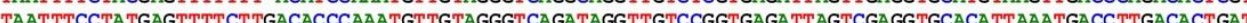

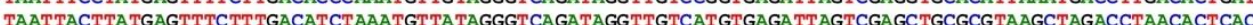
TAATTTCCTACTAGTTTTCTTGACATCTAAATGTTGTAAGGTCAAAAAAGTTTTCTTGTGAGATTAGGCGAGGTGCATATAAGTTGGCCTGGACACTCAC TAATTTCCTACGAGTTTCCT TGACACTCAAATACTGTAGGGTTA GATGGGTTGTCCT GTGAGATTAGTCGA GA TGCGCG TAAGCTGGCCCGGACACTCAC TAATTTTCTATGAGTTTCCTTGACAGCCAAATGTTGTAGGGTTAGGCGGTTGTTCCGTGAGATTAGTTGAGGTTCGCGTAAGCTGGCCCGAACACTCA TAATTTCCTACGAGTTTCCTTGACACCCAAATGTTGTAGGGCTAGGCGGGTTGTCTCGTGAGA TTAATCGAGGTGCGAGTAAGTTGGCCCGA CACTCG TAATTTTTTACGAGTTACCTTAACACCCAAATGTTGTAGAGTTAGGCGGGTTGTCCCATGAGATTAGTCGAAGTACGCGTAAGCTGACCCGGACACTCAC TAATTTCCTATGAGTTACCTTGACACCAAAATGTTGTGGAGTTATGTGGGTTGTCCCATGAGA TAAGTCAAGATGCGAGTATGCTGGCTTGGACACTCA TAAT TTCTTACAAGTTTTCT TGACACTCAAATGT TGTAGGGTCA GACGGGTT-_-GTCTTGTCGAGTGCACG TAAGTTGGCCCAAACACTCAC TAATTTTCTATTAGTTTTCTTGACACCCTAATGTTGTAGGGTCAGACGAGT_-_-TGTCAGATTAGTTGAGGTGCGATAAGCTGGTTCGAACACTCAC TAATTTCCTACGGGTTTCCTTGACATTCAAATGTTATAGGGTCAGGCGGGTTGTCCTGTGAGATTAGTTAAAG TGTGCGTAAATTGGTTCGAACACTCAC TAATTCCTCA--AGTTTTCTTGACATCCTAATGTTA--GGGTCAAGTGGGTTGTCTCATGAGATTAGTTGAGGTGCAT--AAACCGGTATGGACACTCAC TAATTTCCTACTAGTTTCTTTGGCACCCAAATGTTATAGGGTCAAGAGGGTCATCCCATGAGAGTGG---AGGTGAGTGTAAGCGGCTCAAACACTCA TAATATTATATGAATITCATAGACACCCAAATATTGG-ATATCAGACGGGTTGTCATGTGATATTAATCGAAGTGTATATAAGTTGATCAGAACACTCG: TAATTTCCTACGAGTTTCCTTGACATCCAAATGT TATAGGGTCAGACGAGTTGTCCCGTGAGATTAGTCGAGGTGTGCGTAAGCTGGCCCGGACACTCAC TAATTTCCTATGAGTTTCCTTGACACCCAAAAGTTGTAGGGTCAGATGGGTTGTCCCGTGAGATTAGTCAAGGTGCGCGTAAGCTGGCCCAGACACTCAC TAATTTCCTACGAGTTTTCTTGACAACCAAATGTTGTAGGGTTAGGCGGGTTGTCCGGTGAGATTAGTCGAGATACGCGTAAGCTGGCTCAGACACTCAC TAATTTCCTACGAGTTTCCTTGACACGCAAATGTTGTAGGGTCAGGCGAGTTGTTCCGTGAGA TTAGTTGAGGTGCACATAAGTTGGCCCAGACACTCAC TAATATCTCATGAGTTTCCTTGACACTCAAATGTTGTAGGGTCAGACGGGTTGTCCCATGAGATTAGTTGAGATGCACGGTAGCTGATCTAGACAGTTA TAATTTTCTATGAGTTTTCTTAACACCCAAATGTTGTAGGGTCAAAAAGTTGTCCTGTGTGTTTPGTCGAGGTACGCT - AAGCTAGCTCGGACACTCAC TAATTTCTTCTGGG

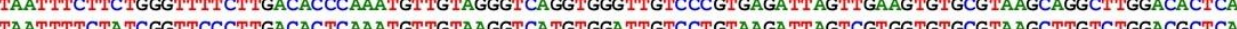
TAM GACACAAATGCCGTGGGGTCTGGCAAGTTGTACCGTGAGATTAATCAAAAGTTGCACAAGCTAGCCCAGACTCTCAC -TGATACCCAATTGTTA TAGCGTAAGACAGATTGTCCGGTGAGATTAGTCTAGGTGCGTGTAAGCTTGCTTGAACACTCAC 1A TAATTTTTTATGAGTTTCCTTAACACCCA -TTGTAATGTCAGGAAGTTGTCCTGTGAGATTAGTCTAGGTGGGTGIAAGATGGCCTGGACACTCG TART TAATTTCTTACGAGTTTTCTTGACACCTAAAAGT TGTAGGTTCAGGCAGGTTATCC- GTGAGATTAGTTGAGGTATGCACAAGTTGGTCTAGACATCCA TAATITCTACGAGTT

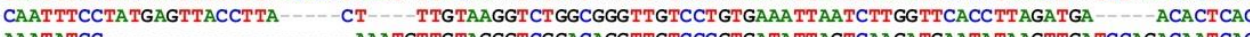
AAATATCC- AAATGTTGTAGGGTCGGACAGGTTGTCCCGTGATATTAGTCAAGA TGAATATAAGTTGATCCAGACAATCAC TAATTICCTATGGGTTACCTTGACACTCAAATATTGTAGGGTCAAGTGGGTTGTCCCGTTAGATTAGTCGAGGTGCGCGTAAACTAGCCCGGACACTAAC TAATTTCCTATGGGTTTCCTTGACACCCAAATGTTGTAGGGTCAGGCGGTTGTCCCGTGAGATTAATCGAGGTGCACA TAAGCTCGTCCGGACAC--AC TAATTTCTTATGGGTTTCCT TGGCGCCCAAATGT TGTAGGGTCAGGCGGTTGTCCCATGAGA TTAGTTGAGGTGCGCGTAAGTTGGCCCAGACACTCA TAATTTCCTACGAGTTTCCTTGACACCCAAATGTTGTAGTGCCAGGTGGATCGTTCAGTGAGA TTAGTTGAAGTGCATGTAAGCTCGCCCGAAAACTTAC TGATTTCCTACGAGTTTCCT TAATACCAAAATGTTGTAGGGTCA GACGGGTTGTCCT GTGAGA TT AGTTTAGGTGCGCG TAAGCTGGCCTTGACATTTA TAAT TTTCTACGAGCTACCTTGACACCCAAATAT TGTAGGGTCTG-CAGGTTGTCCTATGAGACTAGTCGAGGACGTGTAAGCTAACCCGAACACTCA TAATTTCCTACGAGTTTTCTTGACATCCAAATGTTGTAGGGTCAGGTGAGTTGTCCCGTGAGACCAGTAGAGGTGCGCATAAGTTGGTCC-AACACTCA AAATTTTCTATAAGTTTTCTTGATACCCAAATGTTAT--_TC-_CGTGTGATTAGTCGAGGTGCAGGTAATTGGGCGAACACTCAC ATCTCCTACGAGTTTTCTTGATA-TAATTTCCTATGAATTTTCTTGACGTTCATACGTTGTAGAATCAGGTGGGTTATTCAAGGAGATTAATTGAAA TACGTGTAAGCTGATCCGAACATTGA TAATTTCTTACGAGTICCTTGACACCCAAATATTTTGGGTCAAGCGGG -TGTTCTATGAAATTAGTCGAGGTGCGCACAAGCTGGCTCAGACACTCA AAATTTCCTACT AGTTTTCTTGACATCTAAATGTTATCGGGTCAGGCGGGTTGTCCTGTGAGATTAATCGAGGTGTGTGTAAGCTGGTCCGGGCACTTAC TAATTTCCTACGAGTTTCCTTGACACCCAAA-GTTATAGGGTCAAT-_-_TGTTCCATGAAATTAGTCGAGCTATGCGTAAGCTAACTCAAACACTCAC TAAT TTCCTACGAGTTTCCT TGACACCCAAATGT TG TAGGGTCA GGCGGGTTGTCCCGTGAGA TTAGTCGAGGTGCGCGTAAGCTGGCCCGGACACTCAC NAATNTYHHABNRRTTHYHTTgacacBcaAatgtTgtagggT cagDNRDRTYgTccNgtgagA tTAgTHgAgg TgNDNNBWARHTNDNHHNDACANHNR GACAYBCAAATGTTGTAGGGTCAG

Figure 4. MSA of SINE elements of C. sativus. CsSINE-1 primers used for ISAP markers were designed from the conserved regions. The primer site starts from 153 up to 175. 
Table 4. Cucumber genes with inserted CsSINE-1

\begin{tabular}{ll}
\hline Chr. & Gene \\
\hline 1 & Uncharacterized protein LOC101215194 \\
& DNA damage-binding protein 1 \\
& GPI ethanolamine phosphate transferase 3 \\
& Mediator of RNA polymerase II transcription \\
& subunit 13 \\
& Probable ATP-dependent RNA helicase DHX35 \\
& ER membrane protein complex subunit 6 \\
& Histone deacetylase 15 \\
& Protein PIR \\
& MAG2-interacting protein 2 \\
& Translation machinery-associated protein 22 \\
& Transcription initiation factor TFIID subunit 2 \\
Cellulose synthase A catalytic subunit 8 [UDP- \\
forming] \\
E3 SUMO-protein ligase SIZ1 \\
Endoribonuclease Dicer homolog 2
\end{tabular}

2 Nuclear pore complex protein NUP160

Cleavage and polyadenylation specificity factor subunit 1

Uncharacterized protein LOC101217421

Translational activator GCN1

Superkiller viralicidic activity 2-like 2

DEAD-box ATP-dependent RNA helicase 17

WD repeat-containing protein 48

Translation initiation factor eif-2B subunit epsilon

Adagio protein 1

UPF0400 protein C337.03

Gamma carbonic anhydrase 1, mitochondrial

Calcium-transporting atpase 3, endoplasmic reticulum-type

Probable inactive leucine-rich repeat receptor-

like protein kinase At3g03770

Polyadenylate-binding protein RBP47

3 Importin-11

Methyltransferase-like protein 10

Inactive poly [ADP-ribose] polymerase RCD1-

like

Putative ATP-dependent RNA helicase DHX33

Uncharacterized protein LOC101216506

Protein disulfide-isomerase 5-4-like

Diacylglycerol O-acyltransferase 2

Dual-specificity protein phosphatase 12-like

Elongation factor Ts, mitochondrial

Protein VASCULAR ASSOCIATED DEATH 1, chloroplastic

Spastin

Uncharacterized protein At1g04910

Uncharacterized protein LOC 101209700

Uncharacterized protein LOC101220986

TOM1-like protein 2

4 Small $\mathrm{G}$ protein signaling modulator 1

Uncharacterized protein LOC101218523

Sm-like protein LSM4

Putative callose synthase 8

Protein NLP9

Probable sphingolipid transporter spinster homolog 2

AP-4 complex subunit epsilon

Golgin subfamily B member 1
Restin homolog

Phosphoglycerate mutase-like protein 1

Probable cytosolic oligopeptidase A

UDP-N-acetylglucosamine-dolichyl phosphate

$\mathrm{N}$ acetylglucosaminephosphotransferase

MADS-box transcription factor 23

FH protein interacting protein FIP2

Replication factor $\mathrm{C}$ subunit 3

Uncharacterized protein LOC101208689

Phosphoglucan, water dikinase, chloroplastic

5 Transcription factor GTE1

Anaphase-promoting complex subunit 1

DNA repair protein complementing XP-C cells homolog

Uncharacterized protein LOC101212003

Structural maintenance of chromosomes protein 4

Protein decapping 5

Dolichyl phosphate beta-glucosyltransferase-like

Putative nuclear matrix constituent protein 1-like protein

Signal peptide peptidase-like 2

Uncharacterized protein LOC101212864

DNA-directed RNA polymerase V subunit 1

GDP-Man:Man(3)glcnac(2)-PP-Dol alpha-1,2-

mannosyltransferase
6

1

1

2

1

1

1

1

1

1

1

1

1

1

1

1

1

1

1

1

1

1

1

7
1

1

1

1

1

1

1

1

1

1

1

1

1

1

1

1

1

1

1

50S ribosomal protein L20, chloroplastic

DNA-directed RNA polymerase I subunit RPA2

COP9 signalosome complex subunit 3

DNA polymerase epsilon catalytic subunit A-like

Signal peptide peptidase-like 5

Uncharacterized protein LOC101209603

Ankyrin repeat domain-containing protein 2

Uncharacterized protein LOC101204078

Myosin-6-like

Probable plastidic glucose transporter 1

Mediator of RNA polymerase II transcription

subunit 33A

Nardilysin

Uncharacterized protein LOC101216170

DNA gyrase subunit A, chloroplastic/mitochondrial

GPI transamidase component PIG-T

Glyoxylate/hydroxypyruvate reductase HPR3

DEAD-box ATP-dependent RNA helicase 39

Protein FAM188A

Exosome complex exonuclease RRP44

Phosphatidylserine decarboxylase proenzyme 2

HUA2-like protein 3

Brefeldin A-inhibited guanine nucleotide-

exchange protein 1

Ubiquitin thioesterase OTU1

E3 ubiquitin-protein ligase PRT1

Nuclear pore complex protein NUP107

Methylcrotonoyl-coa carboxylase subunit alpha, mitochondrial

Methylmalonate-semialdehyde dehydrogenase

[acylating], mitochondrial

Probable galacturonosyltransferase 6

TBC1 domain family member 15 -like

Pyrophosphate-energized membrane proton pump 2

Nuclear pore anchor

Protein-tyrosine-phosphatase PTP1

Dol-P-Man:Man(5)glcnac(2)-PP-Dol alpha-1,3-

mannosyltransferase 
Protein transport protein SFT2-like

Piezo-type mechanosensitive ion channel homolog

V-type proton atpase subunit a3-like

Calcium-transporting atpase 9, plasma membrane-type

Protein transport protein SFT2-like

Uncharacterized protein LOC101216857

Inositol 1,3,4-trisphosphate 5/6-kinase 4

Uncharacterized protein LOC101209189

Nitrogen regulatory protein P-II homolog

NAD-dependent protein deacetylase SRT1

Uncharacterized protein LOC101216108

Serine/threonine-protein kinase TOR

Pentatricopeptide repeat-containing protein

At2g31400, chloroplastic

Pre-mrna-processing protein $40 \mathrm{C}$

Probable protein phosphatase $2 \mathrm{C} 60$

Uncharacterized protein LOC101208216

Epidermal growth factor receptor substrate 15-like 1

Uncharacterized protein LOC101215677

Alpha-1,3-mannosyl-glycoprotein 2-beta-n-acetylglucosaminyltransferase

Transcription initiation factor TFIID subunit 1

Cycloartenol synthase

DNA polymerase kappa

LOW QUALITY PROTEIN: phospholipase A-2-

activating protein

Exocyst complex component SEC5A

30S ribosomal protein 2, chloroplastic

Dynamin-like protein ARC5

Phosphatidylserine decarboxylase proenzyme 2

$\mathrm{BTB} / \mathrm{POZ}$ domain-containing protein At4g08455

Uncharacterized protein LOC101205573

Ceramide-1-phosphate transfer protein

Probable indole-3-pyruvate monooxygenase

YUCCA10

Sacsin

$\mathrm{N}$-alpha-acetyltransferase 15 , nata auxiliary subunit Indole-3-glycerol phosphate synthase, chloroplastic Calmodulin-binding transcription activator 2

Note: Chr.: Chromosome

Table 5. Summary of ISAP marker analysis

\begin{tabular}{lccccc}
\hline \multicolumn{1}{c}{ Primer name } & Ta $\left({ }^{\circ} \mathbf{C}\right)$ & $\begin{array}{c}\text { Total amplified } \\
\text { bands }\end{array}$ & Band size (bp) & $\begin{array}{c}\text { Total polymorphic } \\
\text { bands }\end{array}$ & $\begin{array}{c}\text { Degree of } \\
\text { polymorphism }(\%)\end{array}$ \\
\hline CmLINE_ISAP1 & 59.4 & 4 & $300-2050$ & 4 & 100 \\
CsSINE1-01 & 53.8 & 19 & $270-1600$ & 19 & 100 \\
CsSINE1-02 & 56.1 & 8 & $250-1200$ & 7 & 87.5 \\
CsSINE1-03 & 60.4 & 10 & $260-1300$ & 14 & 100 \\
SolS-IIIaF & 49.5 & 5 & $300-1600$ & 3 & 60.0 \\
SolS-IVR & 53.0 & 8 & $400-2500$ & 8 & 100 \\
CsSINE1-01/CsSINE1-02 & 60.0 & 15 & $216-1900$ & 14 & 93.0 \\
CsSINE1-01/CsSINE1-03 & 45.0 & 18 & $240-1400$ & 18 & 100 \\
CsSINE1-02/CsSINE1-03 & 45.0 & 9 & $220-620$ & 9 & 100 \\
\hline
\end{tabular}

CsSINE-1 was found to insert into many gene loci in the cucumber genome (Table 4). This result indicates that SINE elements are dispersed all over 7 pairs of cucumber chromosomes. Sixty and 21 copies of CsSINE-1 and CmLINE-1 were retrieved from the cucumber and melon genomes, respectively, for MSA analysis. For ISAP marker development, only highly conserved DNA sequences were used for MSA (Figures 3 and 4).

\section{Amplification of genomic DNA with ISAP marker}

The ISAP primers were designed from the conserved regions of CsSINE-1 and CmLINE-1 (Figure 3 and Figure 4). Six out of seven ISAP primers (single-primer PCR) and three combinations of CsSINE-1 primers generated multiple PCR band patterns when applied on different Cucumis accessions. Single-primer PCR of ISAP primers generally produced 4 to 19 PCR bands, while the combination of CsSINE-1 primers produced 9 to 18 PCR bands. The highest number of PCR bands (19) was generated by a single CsSINE1-01 primer, while the highest number of amplified bands from the ISAP primer combination was achieved by CsSINE1-01/CsSINE1-03 (Figure 5a). All ISAP primers designed from Cucumis species showed higher degrees of polymorphism $(87.5 \%$ -
$100 \%)$ than potato ISAP primers (60\%-100\%) (Table 5). This finding indicates that Cucumis ISAP primers designed in this study have better discrimination power than ISAP primers from Seibt et al. (2012).

The transferability of Cucumis ISAP markers was demonstrated in Coleus species, an ornamental plant that is known for its attractive leaf feature. High degree of polymorphisms was observed among the Coleus accessions, and the amplicon sizes of Cucumis ISAP range from $150 \mathrm{bp}$ to $2000 \mathrm{bp}$ (Figure 6a). Clustering analysis using the UPGMA method showed that the Coleus accessions are clustered into four clusters. Cluster 1 consists of both pink and green leaf accessions (Col-K01, Col-N03, Col-O01, Col-M02, Col-N02, Col-N01, Col-L01, and Col-L02). The second cluster consists of species with pink leaf color (Col-K03 and Col-O02). The third and fourth clusters consist of only one member, namely, ColM01 and Col-K02, respectively (Figure 6b).

\section{Clustering analysis of ISAP marker}

Clustering analysis of ISAP marker was performed on 21 accessions of $C$. melo to investigate their genetic diversity. Pitrat (2008) divided Cucumis melo into two subspecies, i.e. melo and agrestis. The melon accessions 
and the distantly related wild species Cucumis metuliferus were also included in the analysis. The UPGMA dendrogram of ISAP marker separated $C$. metuliferus (US143) from all $C$. melo accessions and provided good discrimination power, with the similarity coefficient ranging from 0.09 to 1.00 (Figure 5b). All 22 melon accessions were grouped into three major clusters. The largest cluster consists of the subcluster of the C. melo group (i.e., Golden Langkawi, Melani, Japonica, NI19, P107, Dainty, Madesta, Haru, Silver Light, Rock Sonya,
US176) and the subcluster of the $C$. agrestis group (i.e., US128, US205, and US 368). However, two accessions of the $C$. agrestis group (US 340 and P34) were clustered together with members of the $C$. melo subcluster. The second cluster consists of Eksis, US58, Baladewa, and US54 originated from the $C$. melo group. The third cluster consists of only $C$. metuliferus (US143). These results suggest that ISAP is a fairly good marker in discriminating Cucumis accessions.
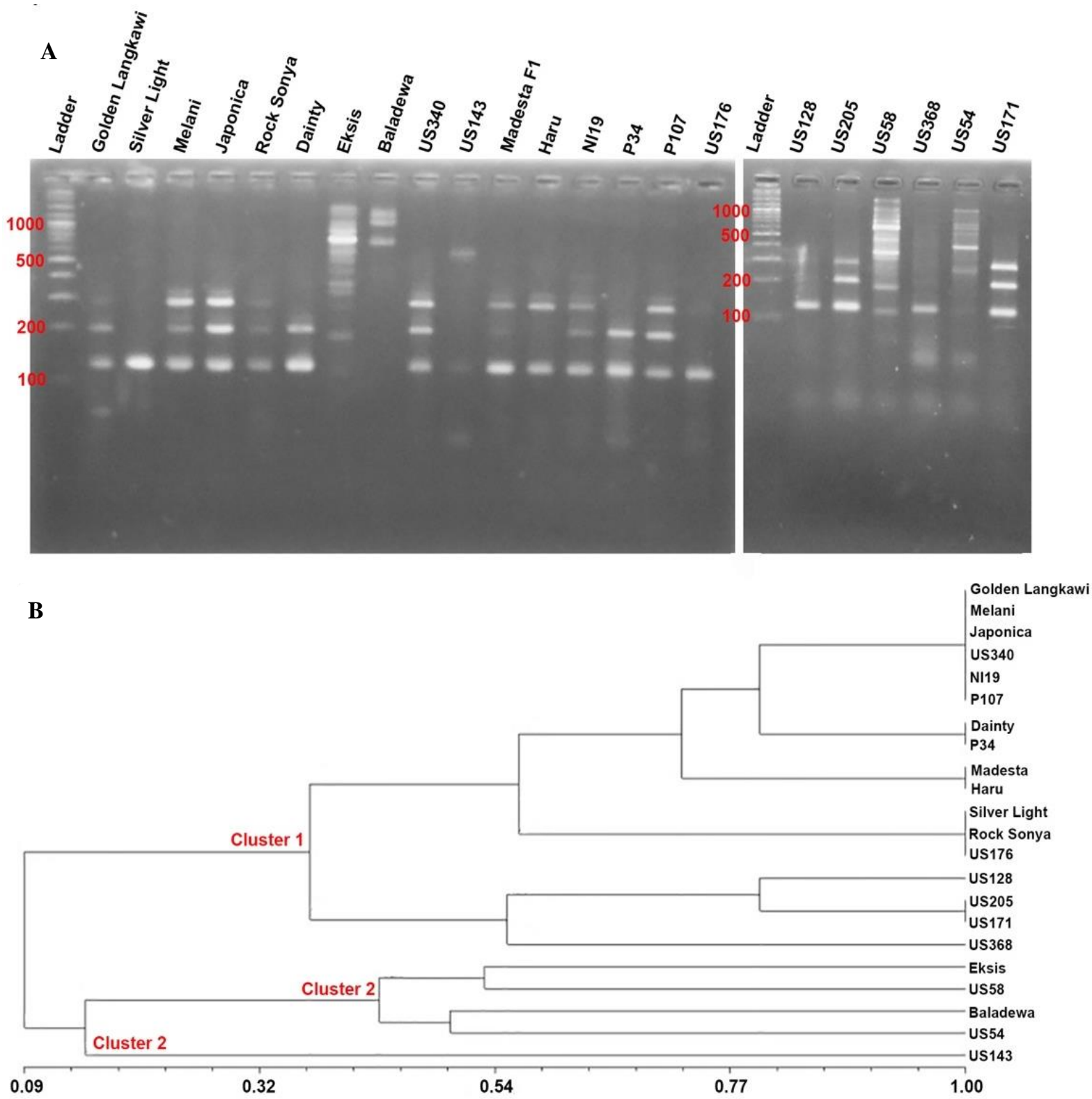

Figure 5. Genomic DNA amplification and cluster analysis of 22 melon accessions. A. ISAP banding patterns generated by the primer combination CsSINE1-01/CsSINE1-03. B. Dendrogram was constructed with the UPGMA clustering method. The scale beneath the dendrogram depicts the genetic similarity coefficient. 
A
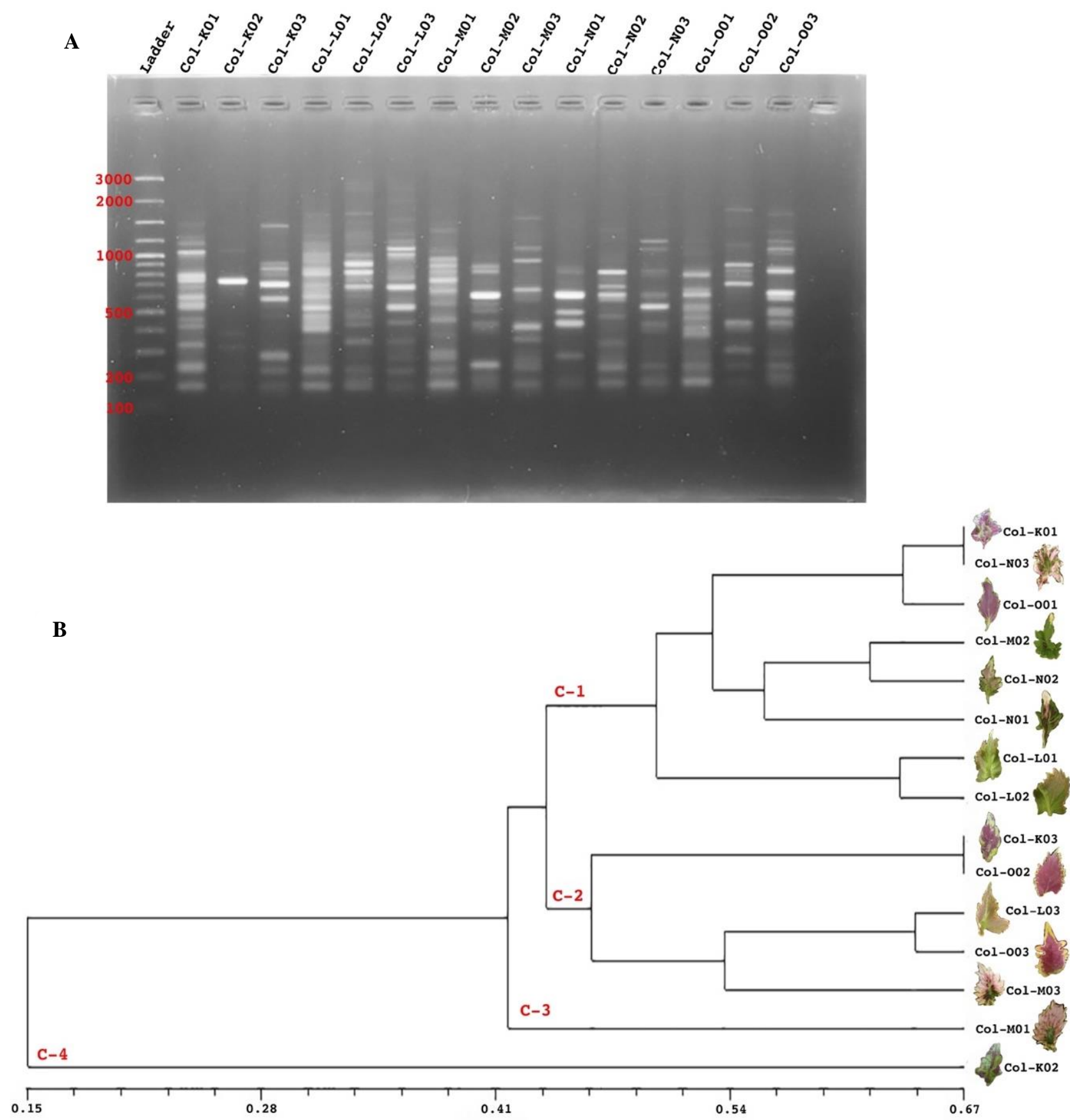

Figure 6. Genomic DNA amplification with ISAP marker and cluster analysis of Coleus accessions. A. ISAP banding patterns of Coleus accessions generated by CsSINE1-01 primer. B. Dendrogram constructed with the UPGMA clustering method among 15 Coleus accessions. The scale beneath the dendrogram depicts the genetic similarity coefficient. C-1, C-2, C-3, C-4 depict cluster 1, 2, 3, 4, respectively.

\section{Discussion}

Both LINE and SINE elements were found in the cucumber (C. sativus) genome (CsLINE-1 and CsSINE-1), whereas only SINE element (CmSINE-1 and CmSINE-2) was found in the melon (C. melo) genome. Multiple copies of these elements were detected in both genomes through homology search analysis. This finding indicates that both SINE and LINE elements are abundant in the melon and cucumber genomes. Non-LTR retrotransposons, particularly LINEs and SINEs, are highly abundant in plant genome, and they have been identified in C. melo (Rodríguez-Moreno et al. 2011; Garcia-Mas et al. 2012; Setiawan et al. 2020b); Brassica rapa, B. napus, B. nigra, B. juncea, B. oleracea, B. carinata (Nouroz et al. 2017, 2018); Solananum tuberosum, Brachypodium distachyon, Populus trichocarpa, Vitis vinifera, Nuphar advena, Medicago truncatula (Wenke et al. 2011), and Lotus japonicus (Gadzalski and Sakowicz 2011). Sequence 
synteny analysis revealed that CmSINE-1, CmSINE-2, and CsSINE-1 originated from CsLINE-1 (Figure 1). This result indicates that SINE elements derived from CsLINE-1 are highly conserved in $C$. melo and $C$. sativus. Weiner (2002) reported that SINEs are similar to LINEs but have a shorter sequence length (100-500 bp), are almost certainly dependent on the activity of LINE RT/EN for their retrotranspositions, and contain polymerase III as their internal promoter instead of polymerase II. SINE is a type of non-autonomous retrotransposon and can increase its copy number through retrotransposition with the help of LINE-encoded proteins where the proteins recognize the $3^{\prime}$ tail of SINE sequences, which is similar to LINE (Liu et al. 2020).

The genome distribution of SINEs in grass plants, such as Oryza sativa, Triticum aestivum, Triticum urartu, Aegilops tauschii, Phyllostachys edulis, Setaria italica, Setaria viridis, Panicum virgatum, Dichanthelium oligosanthes, Sorghum bicolor, and Zea mays showed high-level enrichment in gene-rich regions and the end of chromosome arms (Mao and Wang 2017). In the potato genome, the chromosomal distributions of SINE elements are scattered in all potato chromosomes (Seibt et al. 2012, 2016). In melon, SINE elements are abundant in copy number (Zhang et al. 2019; Castanera et al. 2020; Yang et al. 2020) and are mostly located in the heterochromatic region in the chromosomes (Setiawan et al. 2020b). In addition, SINEs together with other transposable elements are the major component of Citrullus lanatus, Cucurbita argyrosperma, C. pepo, C. maxima, and C. moschata genomes (Guo et al. 2013; Sun et al. 2017; Montero-Pau et al. 2018; Barrera-Redondo et al. 2019). These findings suggest that SINE is highly abundant in plants and is a suitable source for the development of molecular markers in plants.

Our results showed that CsSINE-1 elements are inserted into multiple gene loci in the cucumber genome (Table 4). Non-LTR retrotransposons have been reported to insert into gene loci and are involved in the genome evolution of Cucumis species (Setiawan et al. 2020b) and other plant species such as sunflower (Helianthus annuus) (Nagaki et al. 2015) and banana (Musa spp.) (Čížková et al. 2013). Castanera et al. (2020) reported that transposable elements can have a potential impact on melon genes and affect their phenotype. This result suggests that SINE elements may play an important role in cucumber cell development, genome evolution, and chromosome organization. Further studies are required to investigate the genome evolution and the chromosomal distribution of SINE elements in cucumber accessions together with their closely and/or distantly related species.

The ISAP marker system is designed based on the principle that PCR amplification of sequence regions between two copies of SINE elements using SINE specific primers will generate a multiple banding pattern with a high level of polymorphism (Wenke et al. 2015). High levels of polymorphism were observed when Cucumis ISAP markers were used for genetic diversity study in 21 accessions of $C$. melo (Figure 5 and Table 5). The clustering analysis showed that melon accessions are separated into three clusters (Figure 5b). The Cucumis ISAP marker also allows clear discrimination among Cucumis accessions, particularly in distinguishing the cultivated melon accessions from the wild accessions. Similar findings were observed when ISAP markers were used for genotyping in potato (Seibt et al. 2012; Wenke et al. 2015). The transferability of Cucumis ISAP marker to other plant species was demonstrated in this study when one of the ISAP markers was used in Coleus species (Figure 6). Coleus spp. are ornamental plants and show high variation in their leaf colors and shapes (Shoaib et al. 2020). In addition, the transposable element has been reported as the major driver for different color expressions in ornamental plants (Hsu et al. 2019). The highly polymorphic ISAP banding patterns observed in different Coleus accessions indicate that Cucumis ISAP marker can be applied for genetic diversity study in Coleus species. In addition, ISAP primers described in Seibt et al. (2012) from potatoes were used in this study and showed a high level of polymorphism among Cucumis accessions (Table 5). This finding further supported the transferable nature of ISAP markers in genotyping of plant species. This result is also consistent with the transferable nature of barley IRAP markers in Musa species (Teo et al. 2005). Thus, our studies indicate that the SINE-based markers can be applied directly across different plant species. Further analysis is required to determine the sequence of SINE and/or LINE in Coleus species to confirm the Cucumis ISAP specificity in this species.

In conclusion, the SINE family identified from cucumber and melon genomes can be designed as ISAP markers and can be used for genotyping of Cucumis accessions. This marker can also be applied for genotyping of Coleus accessions. This study demonstrated a simple PCR-based molecular marker system by using a transposable element, namely, the SINE elements.

\section{ACKNOWLEDGEMENTS}

We thank Dr. Kenji Kato (Okayama University, Japan) for providing the Cucumis seed materials and Safitri Kartika Ningrum and Yugo Ardi Saputra for preparing the Coleus spp. materials.

\section{REFERENCES}

Ali-Shtayeh MS, Jamous RM, Shtaya MJ, Mallah OB, Eid IS, Zaitoun SYA. 2017. Morphological characterization of snake melon (Cucumis melo var. flexuosus) populations from Palestine. Genet Resour Crop Evol 64: 7-22. DOI: 10.1007/s10722-015-0329-0.

Barrera-Redondo J, Ibarra-Laclette E, Vázquez-Lobo A, GutiérrezGuerrero YT, Sánchez de la Vega G, Piñero D, Montes-Hernández S, Lira-Saade R, Eguiarte LE. 2019. The genome of Cucurbita argyrosperma (silver-seed gourd) reveals faster rates of proteincoding gene and long noncoding RNA turnover and neofunctionalization within Cucurbita. Mol Plant 12: 506-520. DOI: 10.1016/j.molp.2018.12.023.

Castanera R, Ruggieri V, Pujol M, Garcia-Mas J, Casacuberta JM. 2020. An improved melon reference genome with single-molecule sequencing uncovers a recent burst of transposable elements with 
potential impact on genes. Front Plant Sci 10: 1815. DOI: 10.3389/fpls.2019.01815.

Č́žzová J, Hřibová E, Humplíková L, Christelová $\mathrm{P}$, Suchánková $\mathrm{P}$, Doležel J. 2013. Molecular analysis and genomic organization of major DNA satellites in banana (Musa spp.). PLoS One 8: e54808. DOI: 10.1371/journal.pone.0054808.

Diekmann K, Seibt KM, Muders K, Wenke T, Junghans H, Schmidt T, Dehmer KJ. 2017. Diversity studies in genetic resources of Solanum spp. (section Petota) by comparative application of ISAP markers. Genet Resour Crop Evol 64: 1937-1953. DOI: 10.1007/s10722-0160484-y.

Elbarbary RA, Lucas BA, Maquat LE. 2016. Retrotransposons as regulators of gene expression. Science 351 (6274): aac7247. DOI: 10.1126/science.aac7247.

Endl J, Achigan-Dako EG, Pandey AK, Monforte AJ, Pico B, Schaefer H 2018. Repeated domestication of melon (Cucumis melo) in Africa and Asia and a new close relative from India. Am J Bot 105: 1662-1671. DOI: 10.1002/ajb2.1172.

Gadzalski M, Sakowicz T. 2011. Novel SINEs families in Medicago truncatula and Lotus japonicus: Bioinformatic analysis. Gene 480: 21-27. DOI: 10.1016/j.gene.2011.01.020.

Garcia-Mas J, Benjak A, Sanseverino W, Bourgeois M, Mir G, Gonzalez VM, Henaff E, Camara F, Cozzuto L, Lowy E, et al. 2012. The genome of melon (Cucumis melo L.). Proc Natl Acad Sci 109: 1187211877. DOI: 10.1073/pnas.1205415109.

Guo S, Zhang J, Sun H, Salse J, Lucas WJ, Zhang H, Zheng Y, Mao L, Ren Y, Wang Z, et al. 2013. The draft genome of watermelon (Citrullus lanatus) and resequencing of 20 diverse accessions. Nat Genet 45: 51-58. DOI: $10.1038 / n g .2470$.

Hall TA. 1999. BioEdit: A user-friendly biological sequence alignment editor and analysis program for windows 95/98/NT. Nucleic Acids Symp Ser 41: 95-98.

Hsu CC, Su CJ, Jeng MF, Chen WH, Chen HH. 2019. A HORT1 retrotransposon insertion in the PeMYB11 promoter causes harlequin/black flowers in Phalaenopsis Orchids. Plant Physiol 180: 1535-1548. DOI: 10.1104/pp.19.00205

Kalendar R, Khassenov B, Ramankulov Y, Samuilova O, Ivanov KI 2017. FastPCR: An in silico tool for fast primer and probe design and advanced sequence analysis. Genomics 109: 312-319. DOI 10.1016/j.ygeno.2017.05.005.

Karimi HR, Bagheriyan S, Esmaelizadeh M, Estaji A. 2016. Genetic relationships among melons using RAPD markers. Int J Veg Sci 22: 200-208. DOI: $10.1080 / 19315260.2015 .1008664$.

Kumar S, Stecher G, Tamura K. 2016. MEGA7: Molecular evolutionary genetics analysis version 7.0 for bigger datasets. Mol Biol Evol 33: 1870-1874. DOI: 10.1093/molbev/msw054.

Liu D, Yang J, Tang W, Zhang X, Royster CM, Zhang M. 2020. SINE Retrotransposon variation drives Ecotypic disparity in natural populations of Coilia nasus. Mob DNA 11: 4. DOI: 10.1186/s13100019-0198-8.

Mao H, Wang H. 2017. Distribution, diversity, and long-term retention of grass short interspersed nuclear elements (SINEs). Genome Biol Evol 9: 2048-2056. DOI: 10.1093/gbe/evx145.

Merheb J, Pawełkowicz M, Branca F, Bolibok-Brągoszewska $\mathrm{H}$ Skarzyńska A, Pląder W, Chalak L. 2020. Characterization of Lebanese germplasm of snake melon (Cucumis melo subsp. melo var. flexuosus) using morphological traits and ssr markers. Agronomy 10: 1293. DOI: 10.3390/agronomy10091293.

Montero-Pau J, Blanca J, Bombarely A, Ziarsolo P, Esteras C, MartíGómez C, Ferriol M, Gómez P, Jamilena M, Mueller L, Picó B, Cañizares J. 2018. De novo assembly of the zucchini genome reveals a whole-genome duplication associated with the origin of the Cucurbita genus. Plant Biotechnol J 16: 1161-1171. DOI: $10.1111 /$ pbi. 12860

Nagaki K, Tanaka K, Yamaji N, Kobayashi H, Murata M. 2015 Sunflower centromeres consist of a centromere-specific LINE and chromosome-specific tandem repeat. Front Plant Sci 6: 912. DOI: 10.3389/fpls.2015.00912

Nouroz F, Noreen S, Khan MF, Ahmed S, Heslop-Harrison JSP. 2017. Identification and characterization of mobile genetic elements LINEs from Brassica genome. Gene 627: 94-105. DOI 10.1016/j.gene.2017.06.015.

Nouroz F, Noreen S, Naveed M, Ahmad K, Heslop-Harrison JS. 2018 Identification and insertion polymorphisms of short interspersed nuclear elements (SINEs) in Brassica genomes. Pakistan J Bot 50: $749-756$.
Orozco-Arias S, Isaza G, Guyot R. 2019. Retrotransposons in plant genomes: Structure, identification, and classification through bioinformatics and machine learning. Int J Mol Sci 20: 3837. DOI: 10.3390/ijms20153837.

Pandey A, Ranjan P, Ahlawat SP, Bhardwaj R, Dhariwal OP, Singh PK, Malav PK, Harish GD, Prabhu P, Agrawal A. 2021. Studies on fruit morphology, nutritional and floral diversity in less-known melons (Cucumis melo L.) of India. Genet Resour Crop Evol 68: 1453-1470. DOI: 10.1007/s10722-020-01075-3.

Pantchev I, Aziz S, Sarsu F, Tomlekova N. 2019. Applicability of ISAP, ISSR and SSR markers in tomato breeding programs. Veg Crop Russ 7132: 24-26. DOI: 10.18619/2072-9146-2019-6-24-26.

Pitrat M. 2008. Melon. In: Prohens J, Nuez F (eds). Handbook of Crop Breeding: Vegetables. Springer, New York.

Pitrat M. 2016. Disease resistance in melon and its modification by molecular breeding techniques. In: Ezura H, Ariizumi T, Garcia-Mas $\mathrm{J}$, Rose J (eds). Functional Genomics and Biotechnology in Solanaceae and Cucurbitaceae Crops. Springer Berlin Heidelberg, Berlin.

Raghami M, López-Sesé AI, Hasandokht MR, Zamani Z, Moghadam MRF, Kashi A. 2014. Genetic diversity among melon accessions from Iran and their relationships with melon germplasm of diverse origins using microsatellite markers. Plant Syst Evol 300: 139-151. DOI: 10.1007/s00606-013-0866-y.

Renner SS, Schaefer H, Kocyan A. 2007. Phylogenetics of Cucumis (Cucurbitaceae): Cucumber (C. sativus) belongs in an Asian/Australian clade far from melon (C. melo). BMC Evol Biol 7: 58. DOI: $10.1186 / 1471-2148-7-58$.

Rodríguez-Moreno L, González VM, Benjak A, Martí MC, Puigdomènech P, Aranda MA, Garcia-Mas J. 2011. Determination of the melon chloroplast and mitochondrial genome sequences reveals that the largest reported mitochondrial genome in plants contains a significant amount of DNA having a nuclear origin. BMC Genomics 12: 424. DOI: $10.1186 / 1471-2164-12-424$.

Rohlf FJ. 2009. NTSYSpc: Numerical taxonomy and multivariate analysis: version 2.2. Exeter Software Setauket, New York.

Schulman AH, Flavell AJ, Paux E, Ellis THN. 2012. The application of LTR retrotransposons as molecular markers in plants. In: Bigot $\mathrm{Y}$ (ed). Mobile Genetics Element: Protocols and Genomic Applications, Method in Molecular Biology. Humana Press, London.

Sebastian P, Schaefer H, Telford IRH, Renner SS. 2010. Cucumber (Cucumis sativus) and melon (C. melo) have numerous wild relatives in Asia and Australia, and the sister species of melon is from Australia. Proc Natl Acad Sci 107: 14269-14273. DOI: 10.1073/pnas. 1005338107 .

Seibt KM, Wenke T, Muders K, Truberg B, Schmidt T. 2016. Short interspersed nuclear elements (SINEs) are abundant in Solanaceae and have a family-specific impact on gene structure and genome organization. Plant J 86: 268-285. DOI: 10.1111/tpj.13170.

Seibt KM, Wenke T, Wollrab C, Junghans H, Muders K, Dehmer KJ, Diekmann K, Schmidt T. 2012. Development and application of SINE-based markers for genotyping of potato varieties. Theor Appl Genet 125: 185-196. DOI: 10.1007/s00122-012-1825-7.

Setiawan AB, Teo CH, Kikuchi S, Sassa H, Kato K, Koba T. 2020a. Centromeres of Cucumis melo L. comprise Cmcent and two novel repeats, CmSat162 and CmSat189. PLoS One 15: e0227578. DOI: 10.1371/journal.pone.0227578.

Setiawan AB, Teo CH, Kikuchi S, Sassa H, Kato K, Koba T. 2020b. Chromosomal locations of a non-LTR retrotransposon, Menolird18, in Cucumis melo and Cucumis sativus, and its implication on genome evolution of Cucumis species. Cytogenet Genome Res 160: 554-564. DOI: $10.1159 / 000511119$

Shoaib RM, Ali SI, Metwally SA, Ibrahim MM, Aboud KA. 2020. Phytochemical and molecular analyses of some Coleus cultivars cultivated in Egypt. Bull Natl Res Cent 44: 105. DOI: $10.1186 / \mathrm{s} 42269-020-00358-0$

Sun H, Wu S, Zhang G, Jiao C, Guo S, Ren Y, Zhang J, Zhang H, Gong G, Jia Z, Zhang F, Tian J, Lucas WJ, Doyle JJ, Li H, Fei Z, Xu Y. 2017. Karyotype stability and unbiased fractionation in the paleoallotetraploid Cucurbita genomes. Mol Plant 10: 1293-1306. DOI: 10.1016/j.molp.2017.09.003.

Teo CH, Siang HT, Chai LH, Faridah QZ, Othman YR, Heslop-Harrison JS, Kalendar R, Schulman AH. 2005. Genome constitution and classification using retrotransposon-based markers in the orphan crop banana. J Plant Biol 48: 96-105. DOI: 10.1007/BF03030568. 
Weiner AM. 2002. SINEs and LINEs: The art of biting the hand that feeds you. Curr Opin Cell Biol 14: 343-350. DOI: 10.1016/S09550674(02)00338-1.

Wenke T, Dobel T, Sorensen TR, Junghans H, Weisshaar B, Schmidt T. 2011. Targeted identification of short interspersed nuclear element families shows their widespread existence and extreme heterogeneity in plant genomes. Plant Cell 23: 3117-3128. DOI: $10.1105 /$ tpc. 111.088682

Wenke T, Seibt KM, Döbel T, Muders K, Schmidt T. 2015. Inter-SINE Amplified Polymorphism (ISAP) for rapid and robust plan genotyping. Batley J, (ed). Springer, New York.

Yang J, Deng G, Lian J, Garraway J, Niu Y, Hu Z, Yu J, Zhang M. 2020. The chromosome-scale genome of melon dissects genetic architecture of important agronomic traits. iScience 23: 101422. DOI: 10.1016/j.isci.2020.101422.

Yildiz M, Ekbic E, Keles D, Sensoy S, Abak K. 2011. Use of ISSR, SRAP, and RAPD markers to assess genetic diversity in Turkish melons. Sci Hortic 130: 349-353. DOI: 10.1016/j.scienta.2011.06.048

Zhang H, Li X, Yu H, Zhang Y, Li M, Wang Haojie, Wang D, Wang Huaisong, Fu Q, Liu M, et al. 2019. A high-quality melon genome assembly provides insights into genetic basis of fruit trait improvement. iScience 22: 16-27. DOI: 10.1016/j.isci.2019.10.049.

Zhu H, Guo L, Song P, Luan F, Hu J, Sun X, Yang L. 2016. Development of genome-wide SSR markers in melon with their cross-species transferability analysis and utilization in genetic diversity study. Mol Breed 36: 153. DOI: 10.1007/s11032-016-0579-3. 